\title{
Experimental calibration of the ARA neutrino telescope with an electron beam in ice
}

\section{R. Gaïor ${ }^{a}$, A. Ishihara ${ }^{a}$, T. Kuwabara ${ }^{a}$, K. Mase ${ }^{* a}$, M. Relich $^{a}$, S. Ueyama ${ }^{a}$ S. Yoshida ${ }^{a}$ for the ARA collaboration,}

M. Fukushima ${ }^{b}$, D. Ikeda ${ }^{b}$, J. N. Matthews ${ }^{c}$, P. Motloch $^{d}$, H. Sagawa ${ }^{b}$, T. Shibata ${ }^{e}$, B. K. Shin ${ }^{f}$ and G. B. Thomson ${ }^{c}$

${ }^{a}$ Chiba University, Yayoi-cho 1-33, Inage-ku, Chiba 263-8522, Japan

${ }^{b}$ ICRR, University of Tokyo, 5-1-5 Kashiwanoha, Kashiwa, Chiba 277-8522, Japan

${ }^{c}$ Physics And Astronomy, University of Utah, 201 South, Salt Lake City, UT 84112, U. S. A.

${ }^{d}$ Kavli Institute for Cosmological Physics \& Department of Physics, University of Chicago, Chicago, IL 60637, U. S. A.

${ }^{e}$ High Energy Accelerator Research Organization (KEK), 2-4 Shirakata-Shirane, Tokai-mura, Naka-gun, Ibaraki 319-1195, Japan

f Osaka City University, Sugimoto 3-3-138, Sumiyoshi-ku, Osaka 558-8585, Japan

${ }^{*}$ E-mail: mase@hepburn.s.chiba-u.ac.jp

\begin{abstract}
Askaryan Radio Array (ARA) is being built at the South Pole aiming for observing high energy cosmogenic neutrinos above $100 \mathrm{PeV}$. The ARA detector identifies the radio emissions from the excess charge in a particle shower induced by a neutrino interaction. Such a radio emission was first predicted by Askaryan in 1962 and experimentally confirmed by Saltzberg et al. using the SLAC accelerator in 2000. We performed an experiment, ARAcalTA, using $40 \mathrm{MeV}$ electron beams of the Telescope Array Electron Light Source located in a radio quiet open-air environment in Utah to verify our understanding of the radio emission and the detector responses used in the ARA experiment. We irradiated an ice target with electron beams. Radio signals from the ice target were clearly observed. The coherences, the polarizations and the angular distributions of the radio signals were measured to characterize them. We also performed a detailed simulation of the radio signals and found that the observed radio signals are consistent with the simulation, meaning that our understanding of the radio emission and the detector responses are within the systematic uncertainties of the ARAcalTA experiment.
\end{abstract}

35th International Cosmic Ray Conference - ICRC2017

10-20 July, 2017

Bexco, Busan, Korea

* Speaker. 


\section{Introduction}

Ultra high energy cosmic rays (UHECRs) with the energies above $5 \times 10^{19} \mathrm{eV}$ have been measured by ground-based detectors such as Pierre Auger and Telescope Array (TA), but their origin has not yet been revealed completely. UHECRs interact with $3 \mathrm{~K}$ cosmic microwave background photons during their propagation to the Earth and generate cosmogenic neutrinos [1, 2, 3]. Neutrinos rarely interact with matter, so that they can reach the Earth even from the deep Universe. Therefore, the neutrino flux we observe at the Earth reflects the history of cosmic-ray source evolution from the beginning of the Universe up to now, so that the observation of cosmogenic neutrinos can elucidate the origin of UHECRs.

IceCube [4] has performed searches for cosmogenic neutrinos with energies above $100 \mathrm{PeV}$ by observing Cherenkov light derived from secondary relativistic charged particles initiated by neutrino interactions [5], but has not observed cosmogenic neutrinos yet, indicating a mild source evolution consistent with the star formation rate or a heavier composition than protons. Askaryan Radio Array (ARA) is being built at the South Pole to extend the ability to detect cosmogenic neutrinos with about one order of magnitude higher sensitivity [6], aiming for the first detection.

ARA aims to observe radio signals generated through a neutrino interaction in the Antarctic ice for the neutrino detection. The radio signal is the optimal signal to detect such high energy neutrinos because the radio signal overwhelms the optical signals for such high energy neutrinos due to the signal amplification by a constructive interference known as Askaryan effect [7] and the longer attenuation length for the South Pole ice $(\sim 1 \mathrm{~km})$ compared to the optical $(\sim 100 \mathrm{~m})$. The Askaryan signals have been measured by several experiments using accelerators $[8,9,10]$ and probably by air showers [11, 12]. Since the understanding of the radio emission and the detectors is crucial especially for the determination of the energy scale of the ARA experiment, we also performed a similar experiment of the ARA calibration with the TA Electron Light Source (ARAcalTA) using the Telescope Array accelerator installed for their calibration purpose [13] to understand better the radio emissions and the antenna characteristics used for the ARA experiment.

\section{The ARAcalTA experiment}

The ARAcalTA experiment was performed using the TA-ELS $40 \mathrm{MeV}$ electron beams at the TA site in the middle of the desert in Utah in January, 2015. The configuration was set to reproduce the actual configuration in the ARA experiment as close as possible. The schematic of the ARAcalTA experiment configuration is shown in Fig. 1.

TA-ELS ejects electrons upward to emulate air showers for the TA calibration purpose. A block of ice with the size of $100 \mathrm{~cm} \times 30 \mathrm{~cm} \times 30 \mathrm{~cm}$ was placed on top of the beam end pipe as the target to emulate a high energy electro-magnetic (EM) shower. Since the electron energy is lower than the critical energy of $69 \mathrm{MeV}$ in ice, a cascade is not generated in the ice, and electrons stop inside the ice after running about $20 \mathrm{~cm}$ by losing the energy by ionization. Since the track length is limited to $20 \mathrm{~cm}$ in our experiment, the interference is expected to be weaker compared to an actual cascade; however, Askaryan radiation is still expected to be emitted due to a negative charge excess by the electron beam in the ice block. The corresponding EM shower energy is simply estimated by the typical electron number of about $2 \times 10^{8}$ in the beam bunch and 


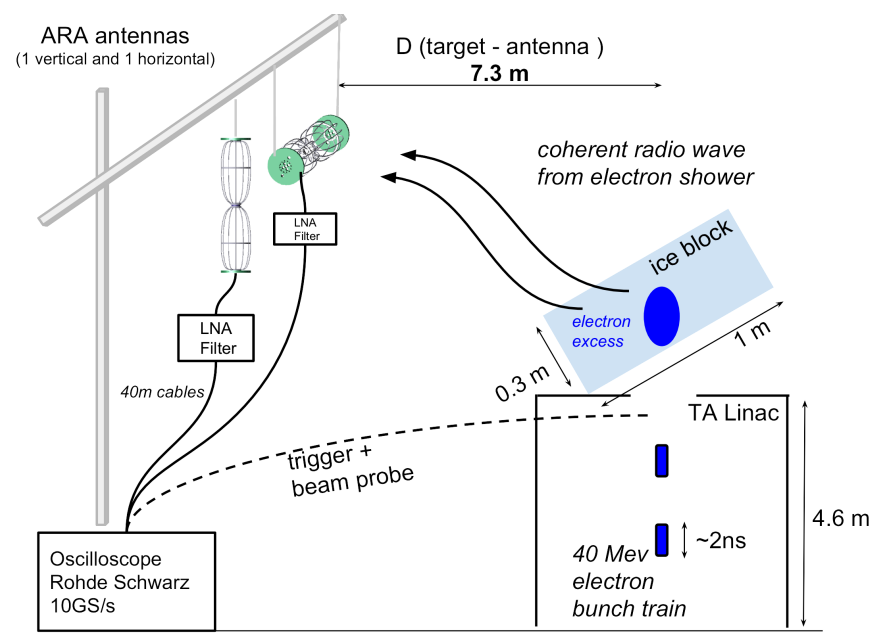

Figure 1: Schematic of the ARAcalTA experiment configuration.

the electron energy of $40 \mathrm{MeV}$, assuming the reasonable negative charge excess ratio of $25 \%$, and is estimated as about $30 \mathrm{PeV}$.

The $40 \mathrm{MeV}$ electron beam from TA-ELS consists of bunch trains that includes about 10 bunches with a time separation of $350 \mathrm{ps}$. The duration time of a bunch train was configured to 1.9 ns (FWHM), down from the value of $1 \mu$ s used in TA, to have the coherence up to high frequency of several hundreds MHz. The rate of the beam was $0.5 \mathrm{~Hz}$ and the maximum electron number in a bunch train is limited to $10^{9}$ by a radiation safety regulation. The electron number was varied during measurements to check the coherence of the radiations by controlling the supply voltage for the accelerator heater. The total electron number was directly measured by a Faraday Cup (FC). Since the FC absorbs all the electrons, it can not be used during measurements using beams. A Wall Current Monitor (WCM), which measures the current on the beam pipe induced by the beam, was used instead to estimate the electron number while radio emissions were observed. A clear correlation between the charge from the FC and the one from the WCM was found, and the uncertainty of the electron number was estimated to be $3 \%$. The lateral beam spread at the bottom of the ice block is estimated to be $4.5 \mathrm{~cm}(1 \sigma)$ by a GEANT4 simulation.

Two ARA birdcage type antennas sensitive to vertically polarized signals were placed vertically and horizontally on top of an antenna tower using an antenna mast to measure the polarization of the signals. An antenna tower was used to measure the angular distribution of the signals, with which the elevation angle from the target to the antenna is accessible up to $45^{\circ}$. The tower was fixed to the ground with the horizontal distance of $7.3 \mathrm{~m}$ away from the beam ejection point to satisfy the far-field condition. The ice target was inclined to angles of $30^{\circ}, 45^{\circ}$ and $60^{\circ}$ to access different emission angles inside ice by making use of a refraction at the top surface of the ice block as shown in Fig. 1.

The observed signals were then filtered by band-pass filters (230-430 MHz) and amplified by low noise amplifiers (LNA) with the gain of $35 \mathrm{~dB}$. This filter is not used for ARA but only for the ARAcalTA experiment to filter environmental noise at the TA site. The signals were transferred 
with $40 \mathrm{~m}$ cables to a fast oscilloscope $(10 \mathrm{GSa} / \mathrm{s})$ to be digitized.

We performed the experiment over two weeks using the accelerator in 8 days in total. The measurements were performed during the day so as not to disturb the TA fluorescence observation. We took data for each configuration of the ice inclination angle and each height as well as the one without any target. Each measurement contains typically 200 events.

\section{Expected signals and the simulation}

Askaryan radiation is expected to be emitted by the electron beam inside the ice target in the ARAcalTA experiment. The radiation is typically expected to show high coherence, a high polarization and a sharp angular distribution peaking at the Cherenkov angle $\left(56^{\circ}\right)$. However, a relatively wide angular distribution is expected for our experiment because the interference condition is weak due to the short electron track $(\sim 20 \mathrm{~cm})$ compared to the wavelength $(\sim 1 \mathrm{~m})$, and the peak is also shifted to vertical direction with respect to the beam direction because the effective track length is longer when looking the beam from a side [14].

Two other components are expected. One is transition radiation (TR) emitted from the boundary between air and ice. Another radiation is from a sudden appearance of the electron beam itself, similar to the expected coherent radio emission from the cosmic ray air shower sudden death [15]. The similar angular distribution peaking at the Cherenkov angle is expected for the TR, while the angular distribution peaks in the almost forward region $\left(0^{\circ}\right)$ for the sudden appearance signals.

Although three radiations are emitted from three different points or a region, they can be treated uniformly by the classical EM theory of the Liénard-Wiechert potentials. The characteristics for all three emissions are similar, having a high polarization and a high coherence.

We mainly used the ZHS method [16] to simulate the electric filed. We also used end-points method [17] to verify our simulation and both methods produced the same results for the case without the ice target.

We developed our simulation based on GEANT4 by including all the accelerator configurations such as the beam energy, the bunch structure and the lateral spread. The electric field has been calculated with the above methods using Liénard-Wiechert potentials. A complication comes from refractions and reflections inside ice. We performed detailed ray trace simulations that takes those effects into account as shown in Fig. 2. The detector responses of the antenna and other components such as band-pass filters, LNA and cables are included to simulate measured waveforms as shown in Fig. 3.

\section{Results}

Clear radio signals were observed for all the configurations with and without the ice target for the vertically placed (Vpol) antenna, while very weak signals were observed for the horizontally placed (Hpol) antenna, indicating the high polarizations of the observed radio signals. An example of the waveform averaged over the 200 events is shown in Fig. 3 for a configuration of the ice inclination angle of $30^{\circ}$ and the antenna elevation angle of $9^{\circ}$. The corresponding simulated waveform is also shown in the figure and shows a reasonable agreement with the observed data. The frequency spectra for the waveform both for data and simulation are also shown in Fig. 3. The 

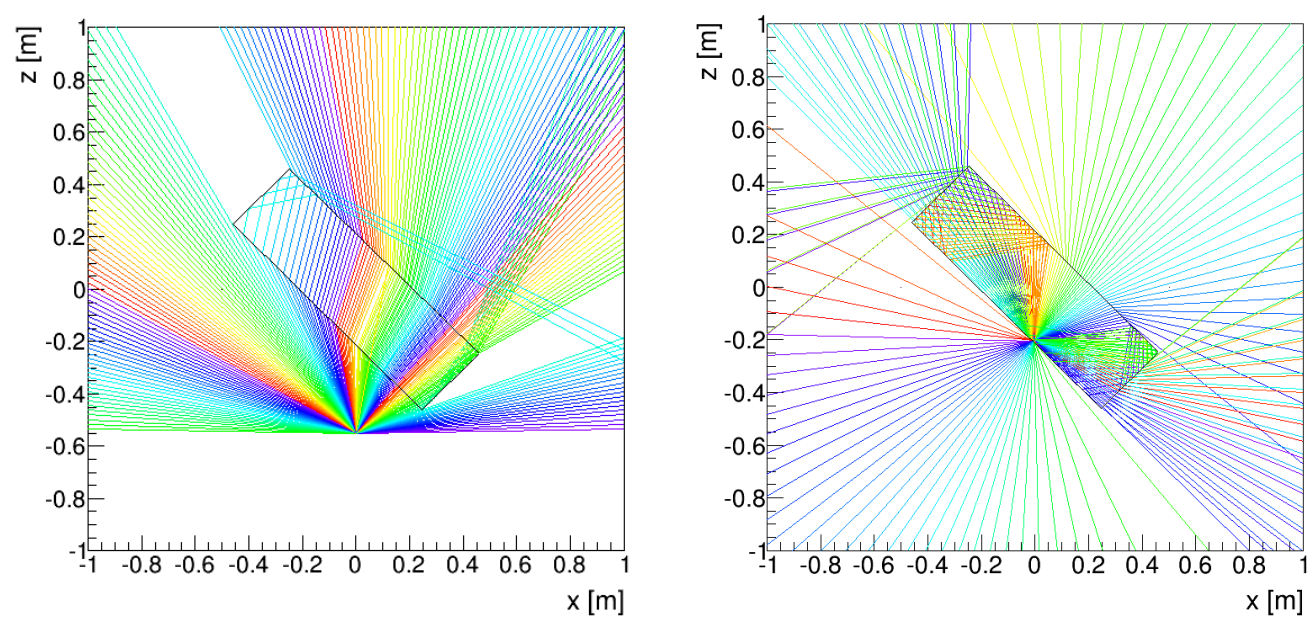

Figure 2: Examples of ray trace simulations that take refractions and reflections into account. Emissions at outside (left plot) and inside (right plot) of the ice block, that correspond to the cases for the sudden appearance signal and Askaryan signal respectively.
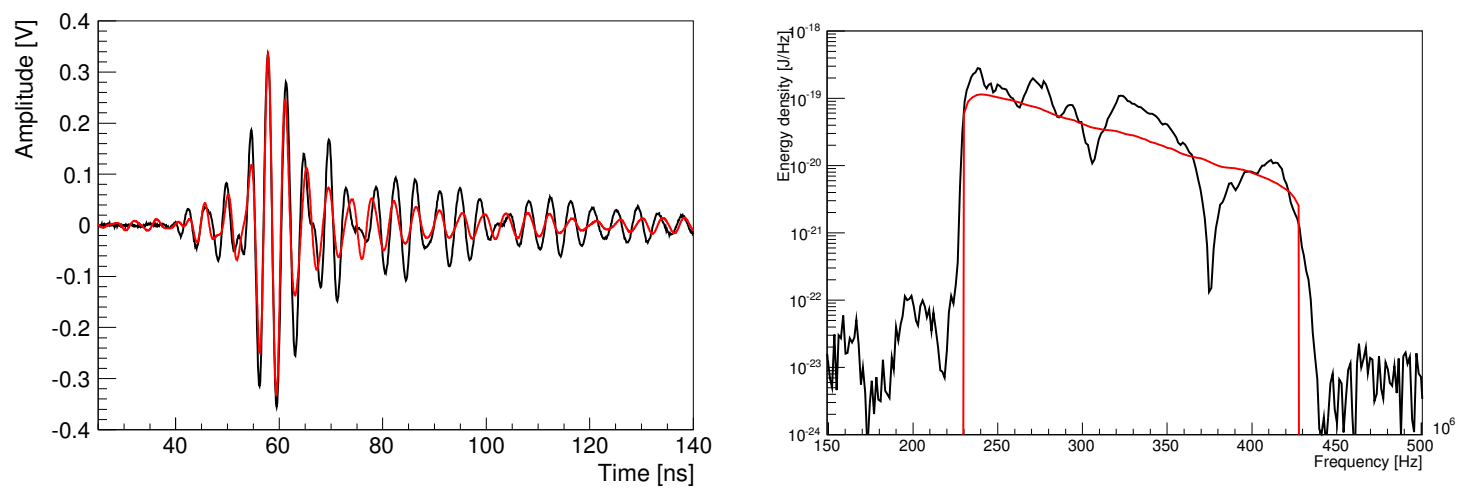

Figure 3: Left: Averaged waveform measured by the vertically polarized antenna with a configuration of the ice inclination angle of $30^{\circ}$ and the antenna elevation angle of $9^{\circ}$ (black) and a simulated corresponding waveform for the configutaion (red). Right: The corresponding frequency spectrum. The sharp cutoffs are due to the band-pass filter to filter environmental noise.

agreement is overall reasonable, although data shows some dips and bumps, indicating the presence of a noise.

Clear radio signals, generated by the sudden appearance of the beams, were measured with a configuration without any target. The signal amplitude is smaller in the case with the ice target, indicating additional radio emission from the ice target.

The polarizations of the signals were first investigated using the Vpol and Hpol antenna signal information to understand the property of the measured signals. High vertical polarizations of 0.92 \pm 0.03 were found in all observed signals for the configuration with the ice target with the angle as expected. The polarization for the configuration without the target was lower - the value of 0.82 \pm 0.03 was found. 


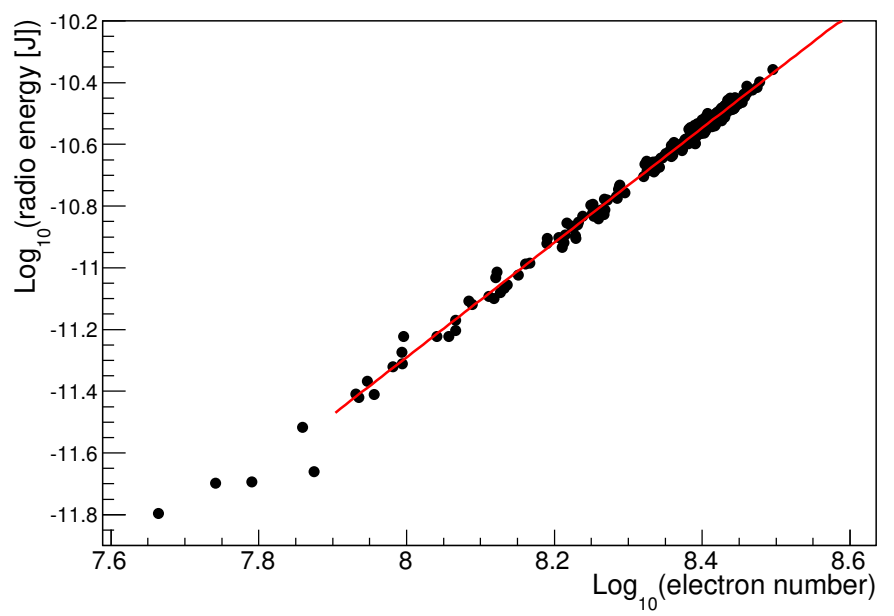

Figure 4: Measured radio power as a function of electron number in the beam for a configuration of the ice inclination angle of $30^{\circ}$ and the antenna elevation angle of $0^{\circ}$. The slope is $1.86 \pm 0.01$, showing a high coherence.

The coherence of the measured signals was investigated next. The signal power is calculated by integrating the square of the waveform amplitude over time. All observed signals with any configuration show a high coherence. The relation between the electron number and radio power for one run is shown in Fig. 4. The high coherence was also found even in the case of no target. The slope index for coherence is derived as $1.78 \pm 0.07$ from all the configurations with Vpol antenna.

Small deviations from the expected values are thought to be due to noise contaminations, and are under investigation.

At last, the angular distributions of measured radio signal energies are plotted as a function of the elevation angle of the antenna for each configuration as shown in Fig. 5. The effect of the noise is minimized using a time window of $10 \mathrm{~ns}$ to extract the main components of the signals. The plot shows clear excesses with the ice block. The reasonable agreements with simulation for all the configurations verify that the emission processes as well as the detector characteristics are understood within the uncertainties.

\section{Systematic uncertainties}

Systematic uncertainties are evaluated and summarized in Table 1.

To check the reproducibility, several data were taken with same configurations. The waveform was stable, keeping the same waveform shape and only showing the amplitude change within 5\%. In order to confirm that our antennas were at the far-field region, the antenna tower was intentionally rotated in azimuthal angle by $\sim 15^{\circ}$. The amplitude of the waveform decreased by $11 \%$ for Vpol antenna after the rotation. The amount of the decrease turned out to be proportional to the geometrical distance change calculated using the signal time delay, showing a behavior at a far-field. The delay time was used to monitor the direction of the antenna mast during the measurements. The spread (rms) of the delay time from the expectation was found out to be $1.9 \mathrm{~ns}$. This corre- 


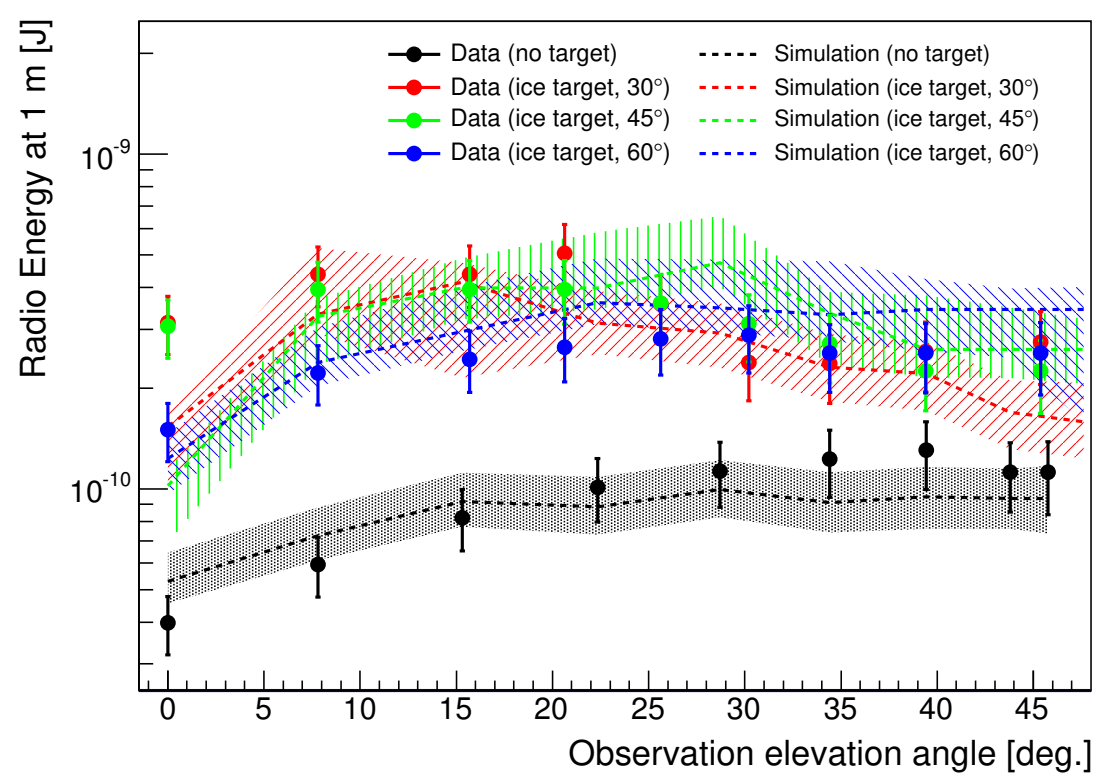

Figure 5: Observed radio signal energy as a function of the observation elevation angle of the antenna for the cases with ice target of the inclination angles of $30^{\circ}$ (red filled circle), $45^{\circ}$ (green filled circle), $60^{\circ}$ (blue filled circle) and without any target (black filled circle). The measured energy is normalized to the typical electron number of $2 \times 10^{8}$. Note that the energy is normalized to the one at $1 \mathrm{~m}$ from the source to correct the geometrical factor. The expected radio signal for each configuration is also plotted with dashed lines with shades to show the one sigma uncertainty (The color notations are as same as measurements).

Table 1: Systematic uncertainties of the ARAcalTA eperiment

\begin{tabular}{c|c|c}
\hline \hline Item & Data & Simulation \\
\hline Statistical error & $\pm 7 \%$ & $\pm 10 \%$ \\
Stability & $\pm 19 \%$ & - \\
Antenna sensitivity uncertainty & - & $+14 \%-17 \%$ \\
Bunch width & - & $+17 \%-14 \%$ \\
\hline Total & $\pm 19 \%$ & $\pm 24 \%$ \\
\hline \hline
\end{tabular}

sponds to a $9^{\circ}$ rotation angle and the distance change by the rotation will change the waveform amplitude by $6 \%$. From these measurements, the systematic uncertainties on the measured radio energy were estimated to be $19 \%$.

The uncertainties for simulation are from the uncertainty of the measured antenna gain and the bunch structure. They are determined from results of measurements in an anechoic chamber and at the site. 


\section{Summary}

We have clearly observed radio signals that show high coherence and high vertical polarization from the ice target using the TA-ELS electron beams. Although we clearly observed radio signals from the sudden appearance of the beams itself, we also observed additional emissions from the ice block. We also performed a detailed MC simulation using GEANT4 by taking the refractions and reflections inside the ice block into account. Reasonable agreement between data and simulation for all the configurations verify that our understanding of the radiation process and our detector is within the uncertainties of the experiment which is $24 \%$, though possible noise components need to be investigated further.

\section{Acknowledgement}

The authors thank to the TA collaboration for the use of the TA-ELS facility and continuous supports to realize the experiment. The authors also thank to Anne Zilles for her kindness to share her source code for the implementation of the ZHS method for our simulation.

\section{References}

[1] V. Berezinsky and G. Zatsepin, Phys. Lett. 28B, 423 (1969).

[2] S. Yoshida and M. Teshima, Prog. Theor. Phys. 89, 833 (1993).

[3] M. Ahlers, L. A. Anchordoqui, and S. Sarkar, Phys. Rev. D 79, 083009 (2009).

[4] M. G. Aartsen et al. (IceCube Collaboration), JINST 12 P03012 (2017).

[5] M. G. Aartsen et al. (IceCube Collaboration), Phys. Rev. Lett. 117, 241101 (2016).

[6] P. Allison et al. (ARA Collaboration), Astropart. Phys. 35, 457 (2012).

[7] G. A. Askaryan, JETP 14, 441 (1962).

[8] D. Saltzberg et al., Phys. Rev. Lett. 86, 13 (2000).

[9] P. W. Gorham et al., Phys. Rev. D 72, 023002 (2005).

[10] P. W. Gorham et al., Phys. Rev. Lett. 99, 171101 (2007).

[11] A. Bellétoile et al., Astroparticle Phys. 69, 50-60 (2015).

[12] A. Aab et al. (Auger Collaboration), Phys. Rev. D 89, 052002 (2014)

[13] T. Shibata et al., Nucl. Instrum. Methods Phys. Res., Sect. A 597, 61 (2008).

[14] R. Gaïor et al., PoS(ICRC2015)1135.

[15] B. Revenu and V. Marin, paper 398, ICRC 2013 proceedings.

[16] J. Alvarez-Muñiz, Phys. Rev. D 81, 123009 (2010).

[17] C. W. James et al., Phys. Rev. E 84, 056602 (2011). 